\title{
Distance Interior Ratio: a new shape signature for 2D shape retrieval
}

\author{
Natsuda Kaothanthong ${ }^{\mathrm{a}, * *}$, Jinhee Chun ${ }^{\mathrm{b}}$, Takeshi Tokuyama ${ }^{\mathrm{b}}$ \\ ${ }^{a}$ Department of Computer Science, Faculty of Science and Technology, Thammasat University, 99 Klongluang, Pathumthani 12120, Thailand \\ ${ }^{b}$ Graduate School of Information Sciences, Tohoku University, Aramaki aza Aoba 6-3-09, Sendai 980-8579, Japan
}

\section{ABSTRACT}

In this work, we propose a shape signature named Distance Interior Ratio (DIR) that utilizes intersection pattern of the distribution of line segments with the shape. To improve the efficiency of the histogram-based shape signature, we present a histogram alignment method for adjusting the interval of the histogram according to the distance distribution. The experimental result shows a $3.25 \% \mathrm{im}$ provement using the proposed histogram alignment. When compared to other shape signatures, our experimental result gives a 77.69\% retrieval rate using MPEG7 Part B dataset (Latecki et al., 2000).

(C) 2016 Elsevier Ltd. All rights reserved.

\section{Introduction}

Shape is an important feature used for describing image content. It can be used in image retrieval, which is the process of identifying images (objects) in a large database that are similar to a given image (Zhang and Lu, 2003) as well as a 3dimensional shape retrieval (Bimbo and Pala, 2006; Liu et al., 2013). Assuming image segmentation has been done and each object in the database is a simple polygon with $n$ vertices, the similarity of the two polygons can be measured using the closest distance between the boundary points on one polygon to the others. Modified Hausdorff distance (Dubuisson and Jain, 1994) and Frenchet distance (Alt and Godau, 1995) are examples of shape similarity measurement. The restriction of such methods is a linear scan of the database when applied in the retrieval system (Shakhnarovich et al., 2006).

A shape signature that is able to characterize the properties of a shape in a compact representation is applied with an efficient search structure, such as Locality Sensitive Hashing (Gionis et al., 1999). A good shape signature should be invariant under rigid motion, and robust to noise and occlusion. There are two main groups of shape signature: region-based methods and contour-based methods (Mingqiang et al., 2008; Zhang and $\mathrm{Lu}, 2003)$. The region-based method considers the information of the pixels within the shape. The shape matrix (Flusser, 1992; Taza and Suen, 1989) is an example of this group. In

\footnotetext{
** Corresponding author: Tel.: +66-2-986-9156;

e-mail: natsuda@cs.tu.ac.th (Natsuda Kaothanthong)
}

contrast, the contour-based method considers the information obtained from the shape contour. Many contour-based methods have been proposed, although the location of the line segment on the shape contour has never been included. Pairwise distance among points on a shape contour (Osada et al., 2002; Ip et al., 2002) or between points and the shape's centroid (Shu and $\mathrm{Wu}, 2011$; Zhang and Wenyin, 2009) are natural information. A normalized histogram is applied in order to achieve a compact size while being invariant to noise and rigid motion. To capture salient features of shape, local quantities such as Shape Context (Belongie et al., 2002), Inner Distance Shape Context (Ling and Jacobs, 2007), and Distance Sets (Grigorescu and Pekov, 2003) can be computed. The signature at each point can be used to efficiently find the correspondence points on the two shapes. Methods such as thin-plate spline (TPS) (Belongie et al., 2002) and dynamic programming (DP) (Ling and Jacobs, 2007) are applied in order to obtain the optimum assignment of correspondence points of the two objects.

Recent local descriptors considered both shape contour and region that are insensitive to articulated shape (Ion et al., 2011) and occluded shape (Janan and Brady, 2015). Both methods apply the geodesic distance in order to find the shortest path inside the shape region to represent a shape's geometric structure. In (De Oliveiria et al., 2015), complex network and spectral graph theories are used to define the shape signature.

\subsection{Motivation and Contributions}

In our preliminary experiment of two-dimensional shape retrieval, we found two problems. The first problem is a homometric pair (Skiena et al., 1990) that is a pair of objects with 


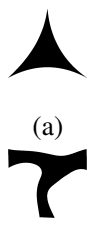

(b)

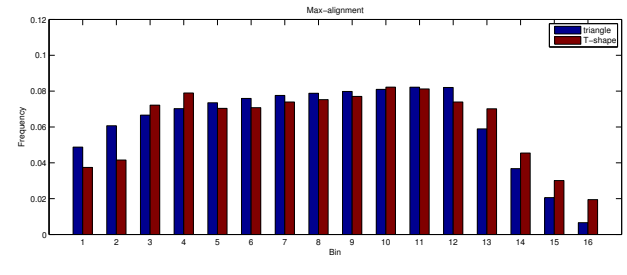

(c)

Fig. 1: Examples of homometric pairs from the MPEG7 partB dataset. (a) A triangle-shape polygon and (b) A T-shape polygon. (c) The distance distributions computed from points on the boundary of polygons (a) and (b). The blue and red colored bars are the distance distribution of (a) and (b) respectively.

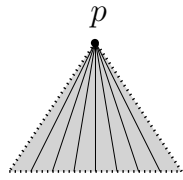

Triangle Shape

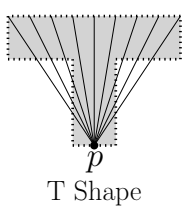

Fig. 2: The line segments defining the distances from a point $p$ to other points on the triangle and the $\mathrm{T}$ shapes.

different shapes but similar distance distribution. See Figure 1 for illustration. The second problem arises when defining the interval of each bin in a histogram using the maximum distance.

According to our observation of the pairwise distance of the homometric pair objects, the distance from a point $p$ to points on the two shapes' contours as in Figure 2 are the same. This is because the pairwise distance distribution does not take into account whether or not the line segment crosses the shape contour. To cope with the homometric pair problem, we proposed a distance called Distance Interior Ratio (DIR) that is able to capture the information of the location of the line segments between the two points on the shape contour. Given a segment $a b$ between two points $a$ and $b$ on the boundary of a region $P$, the DIR (Distance Interior Ratio) of $a b$ is the ratio of the total length of its fragments $P \cap a b$ to the length $|a b|$. Using the DIR distance distribution, the two shapes in Figure 2 are different. The DIR distance of all line segments of a triangle shape in Figure 2 equals 1, while it is strictly less than 1 for some line segments of a T shape.

The proposed DIR distance is different from the inner distance (Ling and Jacobs, 2007) and the geodesic distance (Ion et al., 2011). The inner distance and the geodesic distance consider only the line segments of the shortest path within the shape contour. The inner distance requires a construction of a visibility graph in order to find the shortest path among points on the shape contour. The geodesic distance requires high computation cost when finding the minimum path that joins the two points on a shape contour. Our proposed method considers all line segments that joins two points on a shape contour in order to find the intersection pattern of the distribution of line segments with the shape itself.

To the best of our knowledge, this is the first descriptor that includes the location information of each line segment.

A histogram is a common method that has been used to compress the distribution of brightness of pixels in an image

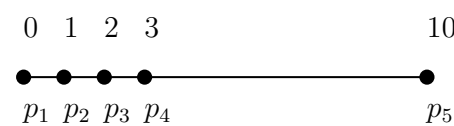

(a) The distance set is $\{1,2,3.10,1,2,9,1,8,7\}$

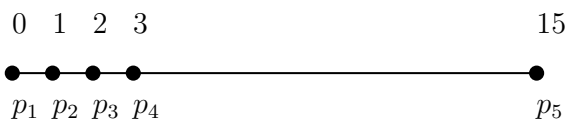

(b) The distance set is $\{1,2,3,15,1,2,14,1,13,12\}$

Fig. 3: Point configurations of two similar point set, which has only one dissimilar point.

(Siggelkow and Burkhardt, 2002) or the distance distributions into a predefined number of bins (Ip et al., 2002; Osada et al., 2002). Usually, the interval is the same for every bin. It is selected as the absolute difference of the minimum and the maximum distance divided by the total number of bins (maximumalignment). Line segments in such a way that the distances are in the interval of each bin is counted.

For such an assignment of line segments to bins, even small changes of either the minimum or the maximum distance may result in shifted bins. Consider the point configurations and their distance sets in Figure 3 (a) and (b). Their maximumalignment histograms are shown in Figure 4 (a) and (b). The interval of the former is 1.8 and the one of the latter is 2.8 . This results in shifted bins. For instance, the line segments of the length 3 switches its bin as depicted in Figure 4 (b). We proposed a new method called mean-alignment. The interval of the bins in the first half of the histogram is defined using the absolute difference between the minimum distance and the mean distance, whereas the interval of the bins in the second half is defined using the absolute difference between the mean distance and the maximum distance. Using the proposed method, the interval of the bins in each half is independently defined using the mean distance. Therefore, small changes of a shape contour result in different histogram in either the first or second half, which represents the bins for short and long line segments, respectively. Figure 4 (c) and (d) illustrate that none of the short line segments in Figure 3 (a) and (b) switches its bin.

Our contributions are a shape signature named "Distance Interior Ratio" (DIR) and a histogram alignment method for adjusting the interval of the histogram according to the distance distribution called "mean-alignment".

Note that, in this research, we are only interested in the efficiency of the shape signature. In other words, no shape matching procedure such as thin-plate spline (TPS) as in (Belongie et al., 2002) and dynamic programming (DP) as in (Ling and Jacobs, 2007) are applied.

\section{Distance Features}

In this work, we consider that the object in the image is segmented and the shape contour is known. We define the shape contour as a set $P=\left\{p_{1}, \ldots, p_{n}\right\}$ where $n$ is the total number of uniformly selected pixels on the contour and $p_{i}=\left(x_{i}, y_{i}\right)$ is 


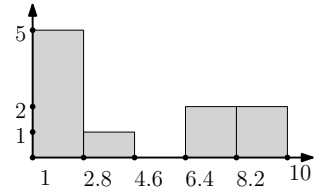

(a) Bin interval is 1.8

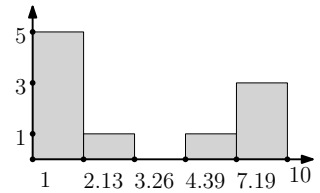

(c) Bin interval of the first half is 1.13 and the other is 2.8 .

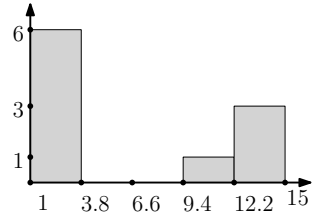

(b) Bin interval is 2.8

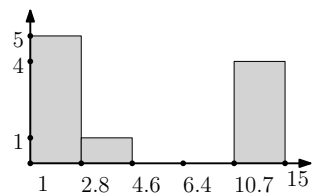

(d) Bin interval of the first half is 1.8 and the other is 4.3
Fig. 4: The bin interval of the depicted maximum-alignment histogram of Figure 3(a) is 1.8 and the one of Figure 3(b) is 2.8. (c) and (d) show the meanalignment histogram of Figure 3(a) and Figure 3(b) respectively.

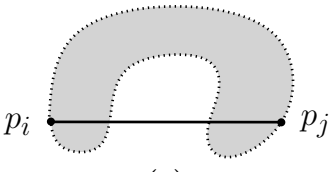

(a)

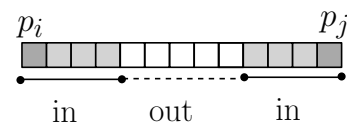

(b)
Fig. 5: (a) A line segment $\left(p_{i}, p_{j}\right)$. (b) The digital line segment, $L\left(p_{i}, p_{j}\right)$, extracted from $p_{i}$ to $p_{j}$. "in" represents the portion of a line within the shape contour while"out" represents the outside portion.

a vector of the coordinate of the pixel $i$. Let $\left(p_{i}, p_{j}\right)$ be a line segment connecting points $p_{i} \in P$ and $p_{j} \in P$ where $i \neq j$.

Two distance features among points in $P$ are used to represent the shape characteristic: the Euclidean distance and the proposed DIR distance. The shape signature of $P$ is a normalized histogram, $H(P)$, that is computed from the feature space, $F$, of the two distance features.

\subsection{Distance Interior Ratio (DIR)}

The DIR distance is the ratio of the summation of portions on a line segment that are lying inside the polygon and the length of the line segment. The detail of the computation is presented.

Let $T(P)=\left\{t_{1,2}, t_{2,3}, \ldots, t_{n-1, n}\right\}$ denotes a set of pairwise DIR distances among points in $P$, where $t_{i j}$ is the DIR distance of $\left(p_{i}, p_{j}\right)$, where $p_{i} \in P, p_{j} \in P$, and $p_{i} \neq p_{j}$. To compute $t_{i j}$, we use Bresenham's Algorithm (Bresenham, 1965) to approximate and extract a digital line segment of $\left(p_{i}, p_{j}\right)$. Let $L\left(p_{i}, p_{j}\right)=$ $\left\{l_{1}, \ldots, l_{k}\right\}$ be a set of $k$ pixels on a digital line. An example of a digital line segment $L\left(p_{i}, p_{j}\right)$ is shown in Figure $5(\mathrm{~b})$. As we only consider binary images, we assume that the intensity $f\left(l_{c}\right)$ of every pixel $l_{c}$ is either 1 or 0 . If the intensity of a pixel $f\left(l_{c}\right)$ equals 1 (dark pixels) then the pixel is lying inside the object's region; otherwise, outside. Let $s_{i j}$ be the number of pixels $l_{c}$ for $1 \leq c \leq k$ with $f\left(l_{c}\right)=1$. From Figure $5(\mathrm{~b})$, we have $s_{i j}=8$. The DIR distance, $t_{i j}$, of a line segment $\left(p_{i}, p_{j}\right)$ is computed using the following formulation,

$$
t_{i j}=\frac{s_{i j}}{k}
$$

Using the above equation, $t_{i j}$ equals 1 if every pixel on the digital line segment is lying inside the shape region as depicted in

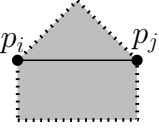

(a)

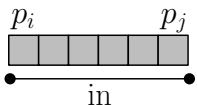

(b)

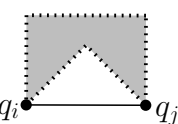

(c)

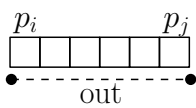

(d)
Fig. 6: (a) A line segment $\left(p_{i}, p_{j}\right)$ is lying inside the shape. (b) The digital line segment $L\left(p_{i}, p_{j}\right)$ that is extracted from (a). The DIR distance of (b) equals 1. (c) A line segment $\left(p_{i}, p_{j}\right)$ is lying outside the shape. (d) The digital line segment $L\left(p_{i}, p_{j}\right)$ that is extracted from (c). The DIR distance of (d) equals 0.

Figure 6 (a) and (b). While it is strictly less than 1 if the digital line segments is partially lying outside and 0 if it is entirely lying outside the shape region as shown in Figure 6 (c) and (d).

The DIR distance is computed for every pair of points $p_{i} \in P$ and $p_{j} \in P$, where $p_{i} \neq p_{j}$, and stored in $T(P)$.

\subsection{Euclidean Distance}

A set of pairwise Euclidean distances among points in $P$ is denoted by $D(P)=\left\{d_{1,2}, d_{2,3}, \ldots, d_{n-1, n}\right\}$, where $d_{i j}$ is the Euclidean distance between points $p_{i}$ and $p_{j}$; and $1 \leq i \leq n-1$, $2 \leq j \leq n$, and $p_{i} \neq p_{j}$. The Euclidean distance between $p_{i}$ and $p_{j}$ is computed as

$$
d_{i j}=\sqrt{\left(x_{i}-x_{j}\right)^{2}+\left(y_{i}-y_{j}\right)^{2}}
$$

The Euclidean distance is computed for every pair of points $p_{i} \in P$ and $p_{j} \in P$ and stored in $D(P)$.

The distance features computed from each pair of points in $P$ is transformed to a feature space $F$, where each line segment $\left(p_{i}, p_{j}\right) \in P$ is represented by the point $\left(d_{i j}, t_{i j}\right)$. More precisely, the $x$-axis represents the Euclidean distances between the points of $P$ and the $y$-axis corresponds to the DIR distance of $P$. The ranges of the $x$-axis and the $y$-axis are $[\min (D(P)), \max (D(P))]$ and $[\min (T(P)), \max (T(P))]$ respectively, where $\min (D(P))$, is the minimum Euclidean distance among points in $P$; $\max (D(P)), \min (T(P))$, and $\max (T(P))$ are defined analogously.

\section{Mean-alignment Histogram}

The shape signature of $P$ is a two dimensional histogram of the DIR and the Euclidean distances that is denoted by $H(P)$. It is computed by counting a number of points plotted on the feature space $F$ that fall into an interval of each bin of a histogram. The interval of each bin is defined according to the mean value of the distance set. Details are presented in this section.

\subsection{DIR Distance Histogram}

Given two positive even integers $c$ and $r$, the feature space $F$ is divided into $c \times r$ bins. The $x$-axis and the $y$-axis are divided into $c$ and $r$ bins respectively. The number of bins are divided into two parts where each part contains $c / 2$ and $r / 2$ bins of the $x$-axis and the $y$-axis respectively. The interval of each part is independently computed according to the mean of the distribution of the Euclidean distance and the DIR distance, i.e., $\mu(D(P))$ and $\mu(T(P))$ respectively. The interval of each bin in the first part on the $x$-axis is equally divided within the range 


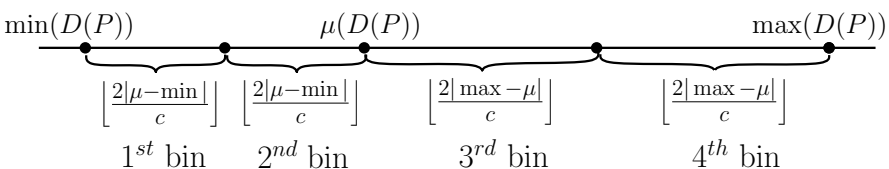

Fig. 7: Interval of each bin of the Euclidean distance histogram.

$[\min (D(P)), \mu(D(P))]$, while the other is $[\mu(D(P)), \max (D(P))]$. The interval on the $y$-axis is defined analogously using the DIR distance.

Let $(u, v)$ denote a bin computed from $F$ where $u$ and $v$ are the index on the $x$-axis and the $y$-axis respectively. The interval of each bin is denoted by $\left[x_{u}, x_{u+1}\right),\left[y_{v}, y_{v+1}\right)$, where the value of $x_{0}$ and $y_{0}$ equals $\min (D(P))$ and $\min (T(P))$ respectively. The interval on the $x$-axis of bins at the index $1<u \leq \frac{c}{2}$ is,

$$
x_{u}=\min (D(P))+\left\lfloor\frac{2|\min (D(P))-\mu(D(P))|}{c}\right\rfloor u
$$

The interval on the $x$-axis of bins at the index $\frac{c}{2}<u \leq c-1$ is computed as follow,

$$
x_{u}=\mu(D(P))+\left\lfloor\frac{2|\max (D(P))-\mu(D(P))|}{c}\right\rfloor\left(u-\frac{c}{2}\right)
$$

The interval of the bins differs according to the distance distribution. From Figure 7, the interval in the second part is wider than the first since most of the line segments on the shape contour are short. The interval of each bin on the $y$-axis is defined analogously using the intervals $\lfloor 2|\min (T(P))-\mu(T(P))| / c\rfloor$ and $\lfloor 2|\max (T(P))-\mu(T(P))| / c\rfloor$.

The DIR histogram $H(P)$ is a $c \times r$ histogram-matrix of $h(u, v)$, where $0 \leq u \leq c-1$ and $0 \leq v \leq r-1$. Let $h(u, v)$ be the number of points that fall into the interval $\left[x_{u}, x_{u+1}\right),\left[y_{v}, y_{v+1}\right)$. $h(u, v)$ is normalized by dividing the total number of points in the feature space. We have,

$$
h(u, v)=\frac{2}{n(n-1)} \sum_{i=0}^{n} \sum_{j=1}^{n-1} g\left(d_{i j}, t_{i j}, x_{u}, x_{u+1}, y_{v}, y_{v+1}\right)
$$

where,

$$
g\left(d_{i j}, t_{i j}, x_{u}, x_{u+1}, y_{v}, y_{v+1}\right)=\left\{\begin{array}{cc}
1, & \text { if } d_{i j} \in\left[x_{u}, x_{u+1}\right) \\
& \text { and } t_{i j} \in\left[y_{v}, y_{v+1}\right), \\
0, & \text { otherwise. }
\end{array}\right.
$$

The two-dimensional DIR histogram of the homometric pair objects are shown in Figure 8 (b) and (d). It can be seen from the histogram that the majority of the line segments in Figure 8

(a) that are within the shape are longer that the ones of Figure 8

(c). See Figure 8 (b) and (d) for illustration.

\subsection{Euclidean Distance Histogram}

From a two-dimensional histogram $H(P)$, we are able to extract a histogram of the Euclidean distance. Let $A(P)=$ $\{a(1), \ldots, a(c)\}$ be the histogram of the distribution of the Euclidean distance, where $a(u)$ is the number of line segments

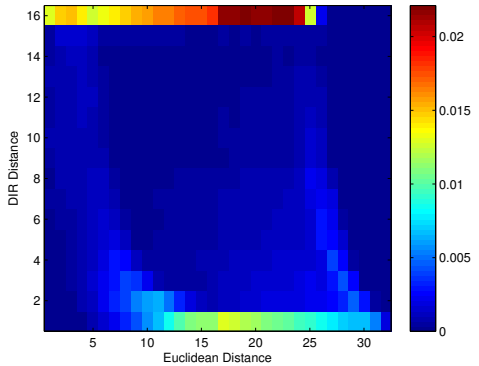

(b) Two-dimensional DIR and Euclidean histogram of the object (a).

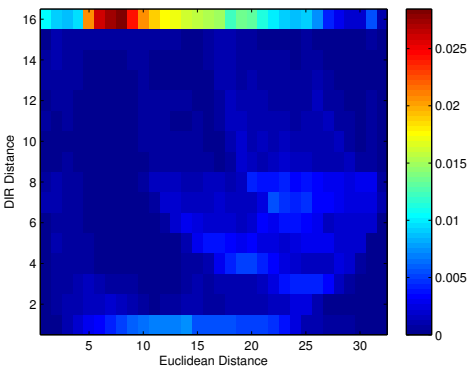

(d) Two-dimensional DIR and Euclidean histogram of the object (c).

Fig. 8: The proposed shape signature of the homometric pair objects.

whose distance falls in the interval of the bin $u^{\text {th }} . a(u)$ is obtained by accumulating the number of lines in every column $v$ at row $u$ of $h(u, v)$. The Euclidean distance of the bin $u^{\text {th }}$ is

$$
a(u)=\sum_{i=0}^{r-1} h(u, i)
$$

Using the above equation to compute the frequency of every bin $u$ for $0 \leq u \leq c-1$, we obtain the mean-alignment Euclidean distance histogram.

\subsection{Histogram Similarity}

Given a polygon $Q$, the pairwise DIR distance $T(Q)$ and the Euclidean distance $D(Q)$, the DIR histogram $H(Q)$, and the Euclidean distance histogram $A(Q)$ are computed as explained in Section 2, Section 3.1, and Section 3.2, respectively.

Let $S(P, Q)$ be the similarity between the histograms of $P$ and $Q$. The similarity of $P$ and $Q$ is the summation of the weighted $L_{1}$ norm of the DIR histogram and the Euclidean distance histogram. We have,

$S(P, Q)=\frac{1}{\alpha}\left(\sum_{i=0}^{r-1} \sum_{j=0}^{c-1}\left|h_{P}(i, j)-h_{Q}(i, j)\right|\right)+\frac{1}{\beta}\left(\sum_{l=0}^{c-1}\left|a_{P}(l)-a_{Q}(l)\right|\right)$

where $\alpha$ and $\beta$ are weight parameters; $h_{P}(i, j)$ and $h_{Q}(i, j)$ are the bin of the DIR histogram at the position $\left(i^{\text {th }}, j^{\text {th }}\right)$; and $a_{P}(l)$ and $a_{Q}(l)$ are bins of the Euclidean distance histogram. From the preliminary experiment, the suitable value for the weight parameters, $\alpha$ and $\beta$, is 2 and 4 respectively. 


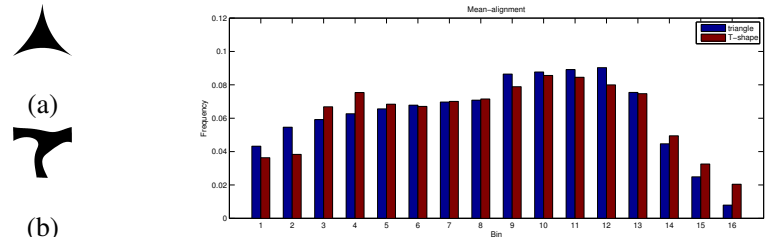

Fig. 9: Comparison of the mean-alignment histogram of the homometric objects (a) and (b).

\section{Characteristic of the Distance Interior Ratio (DIR)}

The mean-alignment histogram is able to represent distance distribution that are partially similar. However, it cannot always give a good representation. The comparison of the meanalignment histograms of the pairwise Euclidean distance of the homometric pairs objects is depicted in Figure 9. The height of the bars at positions 6,7 , and 8 are almost the same. Fortunately, the proposed DIR distance is able to represent the characteristic of each shape differently using the intersection patterns of the distribution of line segments with the shape.

Consider the points on $C_{1}$ and $C_{2}$ in Figure 10. The distance distribution among points of the two sets are the same. Moreover, utilizing the proposed mean-alignment histogram alone could not uniquely represent the histogram. For example, the distance from $p_{3}$ to $p_{4}$ on $C_{1}$ and from $p_{4}$ to $p_{6}$ on $C_{2}$ are the same, i.e. 4. However, considering the location of each line segment, the DIR distance from point $p_{3}$ and $p_{4}$ on $C_{1}$ equals 1 while the DIR distance from point $p_{4}$ and $p_{6}$ on $C_{2}$ is less than 1. Utilizing the location of line segments into the shape signature, the objects, which are homometric, are represented differently.

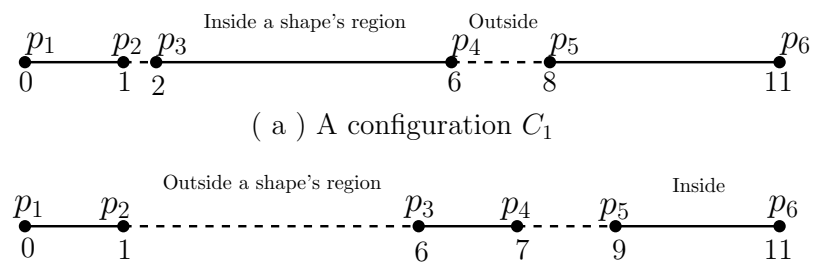

( b ) A configuration $C_{2}$

Fig. 10: Examples of points on a line segment extracted from two different configurations that give the same pairwise distance. The distance set among points on $C_{1}$ and $C_{2}$ are $\{1,1,2,2,3,4,5,5,6,6,7,8,9,10,11\}$.

The DIR histogram is able to represent the geometric structure of a shape. A histogram as depicted in Figure 11 illustrates the relation of the DIR distance and the Euclidean distance. A shape contains a big concavity if majority of long line segments, i.e. line segments in high numbered Euclidean distance bins, belong to low numbered DIR distance bins. Such line segments are contained in bins labeled with "B". On the other hand, a shape is almost convex if most line segments lie in bins labeled with "A".

\section{Experimental Result}

The performance of shape signature using DIR distance is evaluated using the MPEG7 CE-Shape-1 (Latecki et al.,

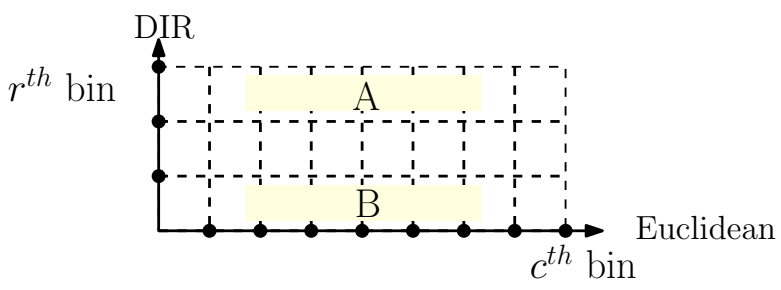

Fig. 11: Some histogram that depicts the DIR distance against the Euclidean distance. The bins labeled with "A" contain the line segments that are (almost) fully contained in the shape. The "B" bins, on the contrary, contain the line segments that barely cross any area inside the shape.

2000) and Kimia's 99 (Sebastian et al., 2004) datasets. For the DIR histogram matrix, we choose $c=\{64,32,16,8\}$ and $r=\{32,16,8\}$. The boundary pixels of each object are extracted and then 500 points uniformly selected. The experiments are conducted on a desktop machine with the Intel Core i5 $2.4 \mathrm{GHz} \mathrm{CPU}$ and $4 \mathrm{~GB}$ of memory. In this research, we are only interested in the efficiency of the descriptor. In other words, no shape matching procedure is conducted on our proposed method.

\section{1. $M P E G 7$}

The MPEG7 CE-Shape (Latecki et al., 2000) dataset consists of 1400 images from 70 classes and each class containing 20 shapes. Examples of object shapes from the MPEG7 Part B dataset are shown in Figure 12. The efficiency of the description is measured using Bull's Eye Percentage (BEP). Every shape signature computed from an image in the dataset is matched with every other shape signature and the top 40 most similar shapes from the same class are counted. So, at most, 20 out of 40 candidates are correct hits.

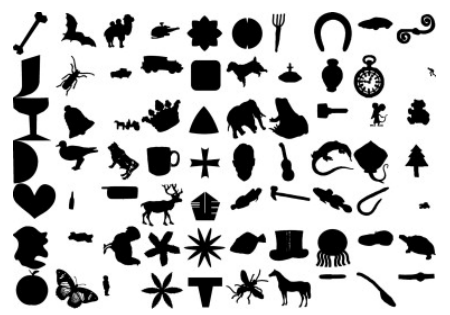

Fig. 12: Example of objects selected from each class from the MPEG9 Part B dataset (Latecki et al., 2000)

\subsubsection{Selecting Suitable Bin Number}

To obtain a suitable number of bin for the Euclidean distance, $c$, and the DIR distance, $r$, we conducted several experiments using the MPEG7 dataset. The retrieval results of using different values of $c$ and $r$ are summarized in Table 1. The size of the matrix that gives the best retrieval result is $c=32$ and $r=16$.

\subsubsection{Efficiency of Histogram Alignment Method}

The comparison of our proposed histogram alignment methods are presented in Table 2. The prefix "max" and "mean" represent the maximum and the mean alignment histogram respectively. The number of bins in the Euclidean distance histogram is $c=32$ and the DIR distance is $r=16$. The results shows that the mean-alignment histogram gives the best 
Table 1: Comparison of the Bull's Eye Percentage (BEP) of the signature computed from DIR distance when using different number of bins of the Euclidean distance histogram $(c)$ and the DIR distance $(r)$.

\begin{tabular}{ccl}
\hline$c$ & $r$ & BEP $(\%)$ \\
\hline \hline 64 & 32 & 77.42 \\
64 & 16 & 77.36 \\
$\mathbf{3 2}$ & $\mathbf{1 6}$ & $\mathbf{7 7 . 6 9}$ \\
16 & 16 & 77.18 \\
16 & 8 & 76.85 \\
\hline
\end{tabular}

Table 2: Comparison of the Bull's Eye Percentage (BEP) of the alignment method. The prefix "max" refers to the maximum alignment histogram and "mean" refers to the mean alignment.

\begin{tabular}{lc}
\hline Method & BEP (\%) \\
\hline \hline max-Euclidean & 54.75 \\
mean-Euclidean & 58.00 \\
max-DIR & 72.40 \\
mean-DIR & 76.60 \\
max-DIR and max-Euclidean & 72.24 \\
mean-DIR and mean-Euclidean & 77.69 \\
\hline
\end{tabular}

result when using either the Euclidean or the DIR distance. The mean-alignment of the DIR distance gives $76.60 \%$ while the maximum-alignment gives $72.40 \%$. The maximum-alignment of the Euclidean distance gives the lowest retrieval rate of $54.75 \%$ while the mean-alignment gives $58.00 \%$. Using the sum of the weighted $L_{1}$ norm of the Euclidean distance histogram and the DIR distance histogram gives $72.24 \%$ for the maximum alignment and $77.69 \%$ for the mean alignment. Examples of the retrieved results are shown in Table 3 . We can see that the mean-alignment DIR distance shape signature is able to retrieve complicated object shapes such as that of a bug.

\subsubsection{Comparison to Other Methods}

The proposed method is compared to the following methods: Shape Context (SC) (Belongie et al., 2002), Inner Distance Shape Description (IDSC) (Ling and Jacobs, 2007), Contour Points Distribution Histogram (CPDH) (Shu and Wu, 2011), Eccentricity Transform (ECCobj2D) (Ion et al., 2011), and Complex Network Spectrum Signature (CNSS) (De Oliveiria et al., 2015). We also compare our result with a SC+TPS and IDSC+DP that applied the thin-plate spline and the dynamic programming in order to find the optimum corresponding points on the two shapes' contours. The results can be found in Table 4. The efficiency of our proposed method is $77.69 \%$, which is higher than all other methods except the IDSC+DP and CNSS.

Although the BEP is $77.69 \%$ for our proposed mean-DIR and mean Euclidean method, we observe that its shape detection ability seems to be higher if we examine typical examples of its incorrectly retrieved results as shown in Table 5.

In the first row, shapes in the class "49" labeled horse are mixed in the query result of an input in the class " 37 " labeled
Table 3: Comparison of the retrieved result using the Euclidean distance and the DIR distance.

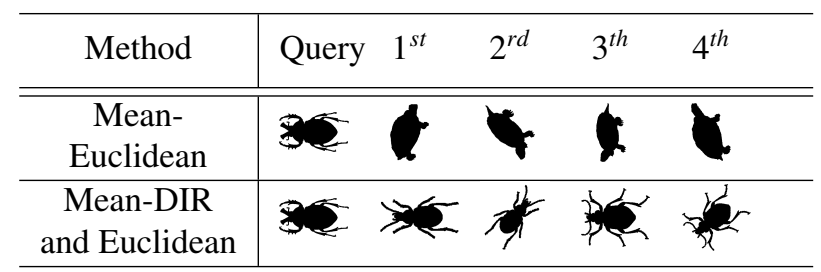

Table 4: Comparison of the Bull's Eye Percentage (BEP) to other shape signature.

\begin{tabular}{lc}
\hline Method & BEP (\%) \\
\hline \hline ECCobj2D (Ion et al., 2011) & 54.56 \\
mean-Euclidean & 58.00 \\
SC (Belongie et al., 2002) & 64.59 \\
IDSC (Ling and Jacobs, 2007) & 68.83 \\
max-DIR and max-Euclidean & 72.24 \\
SC+TPS (Belongie et al., 2002) & 76.51 \\
CPDH (Shu and Wu, 2011) & 76.56 \\
mean-DIR and mean-Euclidean & $\mathbf{7 7 . 6 9}$ \\
IDSC+DP (Ling and Jacobs, 2007) & 85.40 \\
CNSS (De Oliveiria et al., 2015) & 89.47
\end{tabular}

dog. However, both dog and horse are animals with four legs that even human cannot easily separate them. Horses have longer legs, necks, and bodies than dogs, but our classifier could not utilize such subtle quantitative difference; especially when an input query is a shape of a dog with long legs as shown in the figure. The input of the second row is in the class " 20 " labeled chicken. It is wrongly classified as camels (class "15") and elephants (class " 38 "). However, the figure shows indeed a rooster instead of a chicken, and without general knowledge on animals, the geometric shapes are more alike to those two classes. In the third and the fourth rows, it shows that our system is more sensitive to the textures of scars than the shape outline. This is because the texture will affect on DIR much. In the last two rows, we observe that knowledge of the concepts of the labels device0 (class "27") and octopus (class "55") is necessary to correctly classify them.

\subsubsection{Efficiency of Shape Signature}

The proposed shape signature is invariant to rotation and scaling because it applies the distance information and normalizes using the histogram. The results in Table 6 shows correctness of the retrieved objects that are rotated. Moreover, the proposed method is able to represent the complicated shape as shown in the retrieved result of class "64". The objects that are scaled differently are able to retrieve as shown in the Scale Invariance retrieved result in Table 6 . The shape that is occluded can be represented using the DIR shape signature is shown in the Occlusion Invariance retrieved result.

The proposed DIR shape signature has two limitations. The 
Table 5: Examples of retrieved result using mean-DIR and mean-Euclidean. The first image shows the query object.

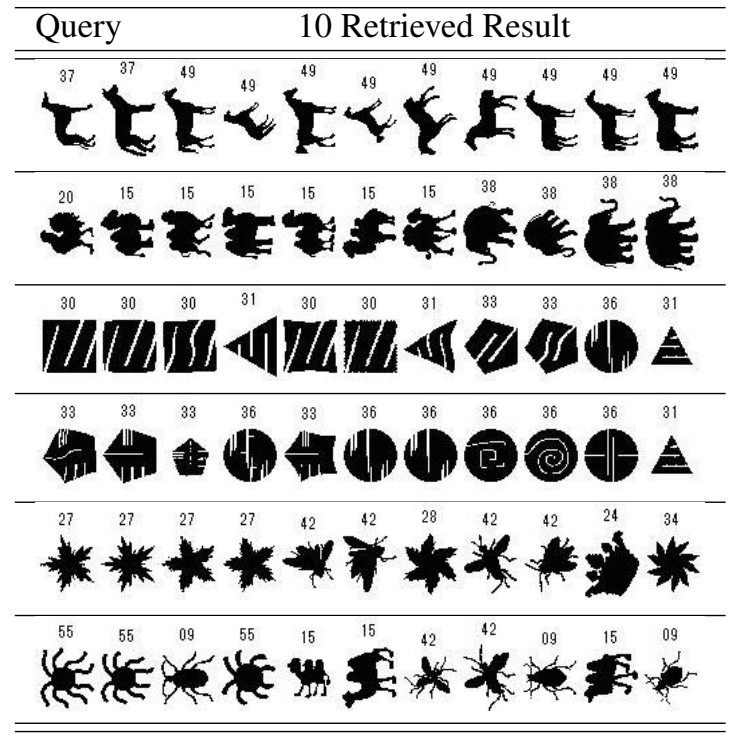

first one is when representing a convex shape object. Since the DIR distance of every line segment of the convex shape equals 1 , only the Euclidean distance is used in a shape descriptor. The result of the convex object from the class " 36 " in Table 6 shows that the retrieved objects are not correct. However, the retrieved shapes have a common characteristic that is the convexity. The retrieved result of the class " 30 " and " 33 " in Table 5 show the result of the convex shapes that have similar patterns. The second limitation is when representing a non-rigid shape transformation. The result of the class " 01 " in Table 6 illustrates the efficiency of the proposed method when retrieving the non-rigid shape. However, the second result of the same class shows bad retrieved results. Comparing the two query shapes, the second one is bended more than the first one.

\subsection{Kimia Dataset}

We compare the recognition accuracy of DIR to the following established method: Shape Context (SC) (Belongie et al., 2002), Inner Distance Shape Description (IDSC) (Ling and Jacobs, 2007), Contour Points Distribution Histogram (CPDH) (Shu and Wu, 2011), Eccentricity Transform (ECCobj2D) (Ion et al., 2011), and the mean-alignment Euclidean (meanEuDist). Recognition is done on the Kimia's 99 shape dataset (Sebastian et al., 2004) consisting of 9 classes, and each containing 11 objects as shown in Figure 13. For each shape, we check whether the 10 closest matches are in the same class as the query object.

The size of the DIR histogram is $r=16$ and $c=16$. A comparison of the recognition rates is summarized in Table 7. Our shape descriptor achieves a higher recognition rate than ECCobj2D and SC+TPS. On the other hand, the recognition rate of our descriptor is lower than the one of the CPDH and IDSC descriptor which applies shape matching after obtaining a set of the best corresponding feature points. Examples of retrieved results using our proposed shape signature are shown in Table 8. The good retrieved results are retrieved objects that are from
Table 6: Examples of retrieved result using the proposed method under rotation, scaling, occlusion, and a convex shape. The first image shows the query object.

Query

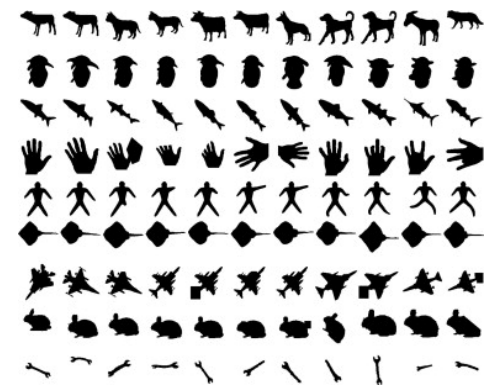

Fig. 13: Objects in Kimia dataset (Sebastian et al., 2004).

the same class as the query. It can be seen that the retrieved results of the occlusion objects such as the query from the class " 09 " and " 06 " are from the same class. Similar shapes from the class " 01 " and " 02 " could not achieve a good result.

\section{Conclusion}

We propose a shape signature that is a two-dimensional histogram of the Distance Interior Ratio (DIR) distance and the Euclidean distance. The DIR distance is computed from the fraction of the portion of a line segment lying inside the shape contour and the length of the line segment. Using the DIR distance, the shape is characterized according to the intersection patterns of the distribution of line segments. If a line segment crosses the shape's concavity part, the DIR distance is strictly less than 1 . The limitation of the proposed shape signature are a convex shape. Since the DIR distance equals 1, only the Euclidean distance is utilized. The other limitation is when representing a non-rigid shape transformation. We also advocate the mean-alignment method as a means to efficiently characterize 
Table 7: Recognition result using Kimia-99 dataset.

\begin{tabular}{l|llllllllll}
\hline Method & $1^{\text {st }}$ & $2^{\text {nd }}$ & $3^{\text {rd }}$ & $4^{\text {th }}$ & $5^{\text {th }}$ & $6^{\text {th }}$ & $7^{\text {th }}$ & $8^{\text {th }}$ & $9^{\text {th }}$ & $10^{\text {th }}$ \\
\hline \hline ECCobj2D & 94 & 85 & 81 & 73 & 81 & 73 & 64 & 59 & 56 & 35 \\
\hline SC+TPS & 97 & 91 & 88 & 85 & 84 & 77 & 75 & 66 & 56 & 37 \\
\hline $\begin{array}{c}\text { mean-DIR } \\
\text { Euclidean }\end{array}$ & 97 & 92 & 89 & 86 & 81 & 80 & 74 & 74 & 70 & 52 \\
\hline $\begin{array}{c}\text { CPDH+ } \\
\text { EMD }\end{array}$ & 98 & 94 & 95 & 92 & 90 & 88 & 85 & 84 & 71 & 52 \\
\hline IDSC+DP & 99 & 99 & 99 & 98 & 98 & 97 & 97 & 98 & 94 & 79 \\
\hline \hline
\end{tabular}

Table 8: Examples of retrieved results mean-DIR and mean-Euclidean using Kimia dataset. The first image shows the query object.

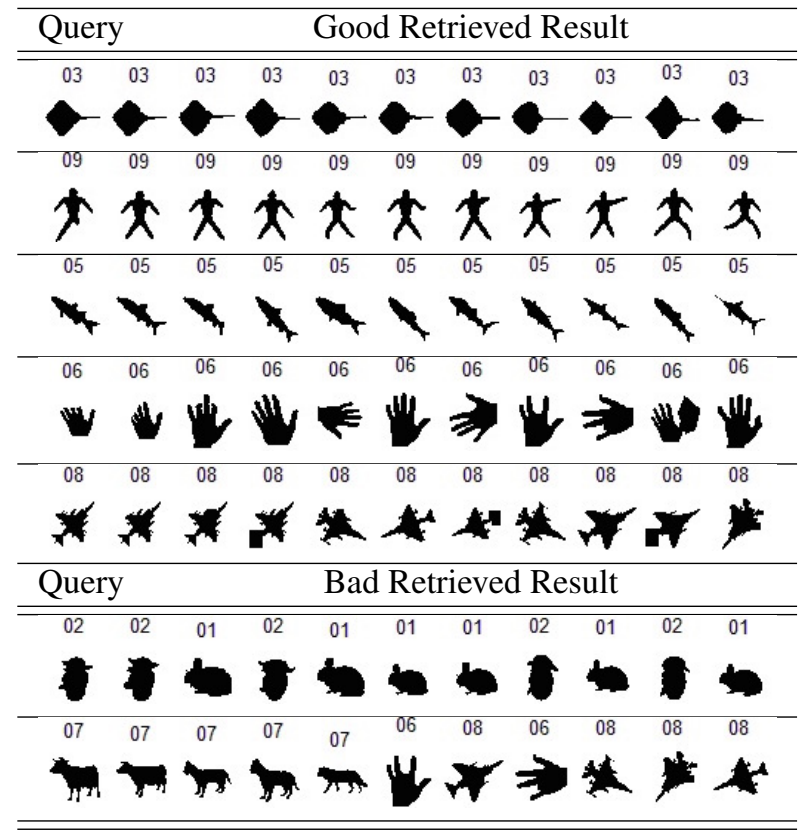

the shape when utilizing the histogram-based shape signature. The experimental result shows the efficiency of the proposed shape signature and the histogram-alignment method. Moreover, the proposed shape signature achieves the highest retrieval rate when the shape matching method such as the dynamic programming is not applied.

\section{Acknowledgement}

The authors would like to thanks the reviewers for their valuable comments. The first author is supported by Faculty of Science and Technology, Thammasat University research fund. The second and the third authors are supported by JST entrusted project "research and development of data integration and analysis for utilization of real-world big data". The second author is also supported by JSPS Grant Scientific Research (C) 25330002. The third author is also supported by JSPS Grant Scientific Research (B) $15 \mathrm{H} 02665$ and Scientific Research for Innovative Area 24106007.

\section{References}

Alt, H., Godau, M., 1995. Computing the Frechet Distance Between Two Polygonal Curves, International Journal of Computational Geometry and Application, 5, pp. 75-91.

Belongie, S., Malik, J., Puzicha, J., 2002. Shape Matching and Object Recognition Using Shape Contexts, IEEE Transactions on Pattern Analysis and Machine Intelligence, vol. 24, No. 24, pp.509-522.

Bimbo, A. D., Pala, P., 2006. Content-based Retrieval of 3D Models, ACM Transactions on Multimedia Computing, Communications, and Application, vol. 2, No. 1, pp.20-43.

Bresenham, J. E., 1965. Algorithm for Computer Control of a Digital Plotter, IBM Systems Journal 4 (1), pp. 25-30.

Chun, J., Kaothanthong, N., Tokuyama, T., 2013. Classified-Distance Based Shape Descriptor for Application to Image Retrieval, Computer Analysis of Images and Patterns, 8048, pp. 1-8.

de Oliveiria, A. B., da Silva, P. R., Couto Barone, D. A. , 2015. A novel 2D Shape Signature Method Based on Complex Network Spectrum, Pattern Recognition Letters, Accepted.

Dubuisson, M.-P., Jain, A. K., 1994. A Modified Hausdorff Distance for Object Matching, Proceeding of International Conference on Pattern Recognition, pp. 566-568.

Flusser, J., 1992. Invariant Shape Description and Measure of Object Similarity, Proceeding of the 4th International Conference on Image Processing and its Applications, pp. 139-142.

Gionis, A., Indk, P., Motwani, R., 1999. Similarity Search in High Dimensions via Hashing, in Proceedings of the 25th International Conference on Very Large Data Basess, pp.518-529

Grigorescu, C., Pekov, N., 2003. Distance Sets for Shape Filters and Shape Recognition, IEEE Transactions on Image Processing, 12, pp. 1274-1285.

Ion, A., Artner, N. M., Peyre, G., Kropatch, W. G., Cohen, L. D., 2011. Matching 2D and 3D Articulated Shapes using the Eccentricity Transform, Computer Vision and Image Understanding, 115, pp. 817-834.

Ip, C. Y., Lapadat, D., Sieger, L, and Regli, W. C., 2002. Using Shape Distributions to Compare Solid Models, Symposium on Solid Modeling and Applications, pp.273-280.

Janan, F., Brady, M., 2015. Shape Description and Matching Using Integral Invariants on Eccentricity Transformed Images, International Journal of Computer Vision, 113, pp. 92-112.

Latecki, L.J., Lakamper, R., Eckhard, U., 2000. Shape Descriptors for Nonrigid Shapes with a Single Closed Contour, IEE Conference on Computer Vision and Pattern Recognition, pp. 424-429.

Ling, H., Jacobs, D.W., 2007. Shape Classification Using Inner-Distance, IEEE Trans. Pattern Analysis and Machine Intelligence, 29(2), pp. 286-299.

Liu, Z., Bu, S., Zhou, K., Gao, S., Han, J., and J. Wu, 2013. A Survey on Partial Retrieval of 3D Shapes, Journal of Computer Science and Technology, 28(5), pp. 836-851.

Mingqiang, Y., Kidiyo, K., Josept, R., 2008. A Survey of Shape Feature Extraction Techniques, Pattern Recognition Techniques, 24-2, pp. 626-664.

Osada, R., Funkhouser, T., Chazelle, B., Dobkin, D., 2002. Shape Distributions, ACM Transaction on Graphics, vol. 21, pp. 807-832.

Sebastian, T. B., Klein, P. N., and Kimia, B. B., 2004. Recognition of Shapes by Editing Their Shock Graphs, IEEE Transaction on Pattern Analysis and Macine. Intelligence, 26(5), pp. 550-571.

Shakhnarovich, G., Darrel, T., Indyk, P., 2006. Nearest-Neighbor Methods in Learning and Vision Theory and Practise, MIT Press, Massachusetts.

Shu, X., Wu, X.J., 2011. A Novel Contour Descriptor for 2D Shape Matching and Its Application to Image Retrieval, Image and Vision Computing , 29, pp. 286-294.

Siggelkow, S., Burkhardt, H., 2002. Improvement of Histogram-based Image Retrieval and Classification, The 16th International Conference on Pattern Recognition, 3, pp. 367-370.

Skiena, S.S., Smith, W. D., Lemke, P., 1990. Reconstructing Sets From Interpoint Distances (extended abstract), The 6th Annual Symposium on Computational Geometry, pp. 332-339.

Taza, A., Suen, C., 1989. Discrimination of Planar Shape using Shape Matrices, IEEE Transaction on Systems, Man, and Cybernetics, 19, pp. 1281-1289.

Zhang, D., Lu, G., 2003. Review of Shape Representation and Description Techniques, Pattern Recognition, pp. 1-19.

Zhang, J., Wenyin, L., 2009. A Pixel-level Statistical Structural Descriptor for Shape Measure and Recognition, The 10th International Conference on Document Analysis and Recognition, pp. 386-390. 